\title{
Cambios en la densidad de recursos humanos en salud durante la epidemia de COVID-19 en el Perú, abril - agosto 2020
} Changes in the density of human resources in health during the COVID-19 epidemic in Peru, april - august 2020

\author{
${ }^{1}$ Observatorio del Sistema de Salud del Perú, Facultad de Medicina, Universidad Nacional Mayor de San Marcos. Lima, Perú. \\ ${ }^{2}$ Grupo de Investigación en Sistemas de Salud SYSTEMIC, Universidad Nacional Mayor de San Marcos. Lima, Perú. \\ ${ }^{3}$ Academia Nacional de Medicina. Lima, Perú. \\ ${ }^{4}$ Unidad de Telesalud, Facultad de Medicina, Universidad Nacional Mayor de San Marcos. Lima, Perú. \\ ${ }^{5}$ Instituto Nacional de Salud. Lima, Perú. \\ ${ }^{6}$ Dirección General de Personal de la Salud, Ministerio de Salud. Lima, Perú. \\ ${ }^{7}$ Facultad de Medicina, Universidad Peruana Cayetano Heredia. Lima, Perú. \\ ${ }^{a}$ Médico cirujano, ORCID: https://orcid.org/0000-0003-3710-2804 \\ ${ }^{b}$ Médico cirujano, doctor en medicina. ORCID: https://orcid.org/0000-0002-2750-1804 \\ ${ }^{\circ}$ Médico especialista en administración en salud. ORCID: https://orcid.org/0000-0003-0293-7107 \\ ${ }^{a}$ Médico especialista en administración en salud. ORCID: https://orcid.org/0000-0001-6922-1213 \\ ${ }^{e}$ Médico especialista en administración en salud, magister en administración. ORCID: https://orcid.org/0000-0001-5727-9100 \\ ${ }^{\dagger}$ Médico especialista en gestión en salud. ORCID: https://orcid.org/0000-0003-4880-2419 \\ ${ }^{9}$ Doctor en estadística matemática, ORCID: https://orcid.org/0000-0002-8051-7549 \\ ${ }^{h}$ Médico cirujano, especialista en informática biomédica en salud global. ORCID: https://orcid.org/0000-0001-6922-1213 \\ Médico cirujano, magister en medicina. ORCID: https://orcid.org/0000-0003-1344-3807 \\ Médico cirujano, magister en política social. ORCID: https://orcid.org/0000-0001-6489-2376 \\ k Ingeniero, ORCID: https://orcid.org/0000-0001-6667-1876 \\ 'Magister en investigación epidemiológica, ORCID: https://orcid.org/ 0000-0001-9518-4241 \\ ${ }^{m}$ Geógrafo, especialista en geografía y medio ambiente. ORCID: https://orcid.org/0000-0001-6433-9538

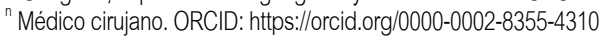

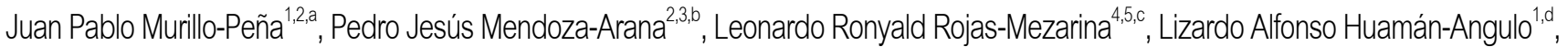
Felipe Peralta Quispe ${ }^{2, e}$, Pedro Antonio Riega-López ${ }^{1, f}$, Manuel Luis Chilca Alva ${ }^{2,9,}$ Javier Silva Valencia ${ }^{4, h}$, Claudia Maria Teresa Ugarte Taboada ${ }^{6, i}$, Javier Cesar Loayza Altamirano ${ }^{6, j,}$ Walter Pérez Lázaro, ${ }^{6, k}$, Miguel Moscoso Porras ${ }^{7,1}$, Arnold Cabana Peceros ${ }^{5, m}$, Stefan Alexis Escobar Agreda, ${ }^{4, n}$

\section{An Fac med. 2021;82(1):5-12. / DOI: https://doi.org/10.15381/anales.v82i1.20822}

\section{Correspondencia:}

Juan Pablo Murillo-Peña

jmurillop@unmsm.edu.pe

Recibido: 21 de junio 2021

Aprobado: 14 de julio 2021

Publicación en línea: 15 de julio 2021

Conflictos de interés: Los autores declaran no tener conflictos de interés.

\section{Fuente de financiamiento:}

Autofinanciado

Citar como: Murillo-Peña J, MendozaArana P, Rojas-Mezarina L, HuamánAngulo L, Peralta F, Riega-López $P$, et al. Cambios en la densidad de recursos humanos en salud durante la epidemia de COVID-19 en el Perú, abril - agosto 2020. An Fac med. 2021;82(1):5-12. DOI: https:/doi. org/10.15381/anales.v82i1.20822
Resumen

Objetivo. Analizar los cambios en la Densidad de Recursos Humanos en Salud(DRHS) en el Ministerio de Salud a nivel regional y provincial en el Perú; a fin de describir la respuesta del sistema de salud sobre este componente durante la epidemia de COVID-19. Métodos. Estudio descriptivo basado en el análisis de la base de datos nacional de INFORHUS del Ministerio de Salud. Para el cálculo de la DRHS, se consideró como numerador al personal de salud registrado en la base de INFORHUS y como denominador a la población adscrita al Seguro Integral de Salud -SIS. Se excluyó a personal de salud que falleció y aún estaba registrado, y a personal diagnosticado de COVID-19 por prueba rápida o molecular durante los meses considerados en el análisis. Seanalizaron los cambios porcentuales de DRHS entre los meses de abril hasta agosto de 2020 a nivel regional y provincial en el Perú. Resultados. En el periodo abril-agosto la DRHS a nivel nacional se incrementó en un 5,1\%. Anivel regional este incremento se logró en 15 de las 25 regiones con una variación desde 0,2\% hasta 12,3\%, y se visualizó una reducción brusca y sostenida del personal nombrado, juntamente con un incremento del personal con contratos temporales. En el resto de las regiones se visualizó una reducción de DRHS la cual varió entre - $-0,7 \%$ hasta $-7,7 \%$. Conclusiones. La DRHS en el Perú mostró un leve incremento a nivel nacional durante el primer periodo de cuarentena por la pandemia de COVID-19; sin embargo, este resultado no ha sido equitativo en las diferentes regiones y provincias a nivel nacional. Esto sugiere una limitación en el alcance de las medidas tomadas por el gobiemo, y la necesidad de tomar acciones para el fortalecimiento de la situación de salud y despliegue de RHUS en los sectores más desfavorecidos.

Palabras clave: COVID-19; Perú; Recursos Humanos; Tendencias; Salud Pública (fuente: DeCS BIREME).

\section{Abstract}

Objective. To analyze the changes in the density of human resources in health (DHRH), in the Ministry of Health at the regional and provincial level in Peru, in order to describe the response of the health system on this component during the COVID-19 epidemic. Methods. Descriptive study based on the analysis of the INFORHUS national database of the Ministry of Health. To calculate the DHRH, the health personnel registered in the INFORHUS database were considered as the numerator and the population assigned to the Seguro Integral de Salud - SIS as the denominator. Health personnel who died and were still registered, and personnel diagnosed with COVID-19 by rapid or molecular testing during the months considered in the analysis, were excluded. The percentage changes of DHRH between the months of april to august 2020 were analyzed at the regional and provincial level in Peru. Results. In the april-august 2020 period, in response to the first phase of the COVID-19 pandemic, Peru increased the DHRH by 5,1\%. At the regional level, this increase was achieved in 15 of the 25 regions with a variation from $0,2 \%$ to $12,3 \%$, and a sharp and sustained reduction in appointed personnel was observed, together with an increase in personnel with temporary contracts. In the rest of the regions, a reduction in DHRH was observed, which ranged from $-0,7 \%$ to $-7,7 \%$. Conclusions. DHRH in Peru showed a slight increase at the national level during the first quarantine period due to the COVID-19 pandemic, however, this result has not been equitable in the different regions and provinces at the national level. This suggests a limitation in the scope of the measures taken by the government, and the need to take actions to strengthen the health situation and deployment of human resources in health in the most disadvantaged sectors.

Keywords: COVID-19; Peru; Workforce; Trends; Public Health (source: MeSH NLM). 


\section{INTRODUCCIÓN}

Para asegurar la efectividad de los sistemas de salud es necesario disminuir los desequilibrios y déficits existentes en la fuerza de trabajo. Con el fin de cuantificar este déficit la OMS ha diseñado la razón de densidad de recursos humanos en salud (DRHS) la cual establece la cantidad mínima de recursos humanos en salud (RHUS) necesaria para responder adecuadamente a la demanda de atención de una cantidad de la población ${ }^{(1)}$. Para el año 2006 este indicador se definió como la disponibilidad de al menos de 23 médicos, enfermeras y parteras por cada 10000 habitantes, umbral que fue incrementado en el 2015 a 44,5 médicos, enfermeras y parteras por cada 10000 habitantes. Este indicador ha sido considerado como referente a nivel mundial por los sistemas de salud para la implementación de estrategias que permitan optimizar la disponibilidad local de los RHUS. A pesar de ello, aún persisten inequidades en su disponibilidad, distribución y calidad entre países y dentro de los países e incluso entre los niveles de atención y los sectores públicos y privados ${ }^{(2)}$.

Por su parte, la pandemia del COVID-19 a nivel mundial ha generado un gran impacto sobre el estado de salud de los RHUS debido a su mayor exposición a esta enfermedad ${ }^{(3)}$. Por ejemplo, al inicio de la pandemia se han reportado cifras de personal infectado desde 3,5 a 29\% en China, 10,7\% en Italia y de 15,6 a 19\% en los Estados Unidos ${ }^{(4)}$ y hasta la actualidad 130 países han reportado fallecimientos de profesionales de salud atribuibles a COVID-19. Del mismo modo, países con una elevada incidencia de casos han mostrado en su personal de salud situaciones de estrés postraumático ${ }^{(5)}$, reportes de suicidios ${ }^{(6)}$ e incluso estigma social ${ }^{(7)}$. Esto representa una seria amenaza para la disponibilidad de RHUS en los sistemas de salud, quienes, ante el contexto la pandemia, deben desplegar un mayor esfuerzo para cubrir con la demanda de atención existente, exponiendo su salud y disminuyendo aún más su disponibilidad.

En Perú la situación de los RHUS presenta limitaciones que los condicionarían a una mayor exposición a la COVID-19 (8) generando una mayor prevalencia de in- fección y contagio por esta enfermedad en esta población ${ }^{(9)}$. Entre las más importantes se señala la irregularidad en la disponibilidad de RHUS vista en el territorio nacional en los últimos 10 años el cual se concentra mayormente en las grandes ciudades y capitales del país a expensas de ciudades menos pobladas y sectores rurales ${ }^{(10)}$. Si bien el gobierno ha implementado diversas estrategias que han permitido acortar estas brechas, la disponibilidad de RHUS aún resulta insuficiente en diversas regiones ${ }^{(11,12)}$, para el logro de las coberturas de aseguramiento ${ }^{(13)}$ y como un problema estructural en el desarrollo de la política pública en salud ${ }^{(14)}$.

Aunque Perú ha mejorado sustantivamente la disponibilidad de RHUS en el sector público (MINSA y gobiernos regionales) a nivel de promedio nacional, no ha modificado de manera sustantiva la equidad de su distribución según territorio, niveles de atención y estratos de pobreza. En el año 2013, la suma de profesionales de medicina, enfermería y obstetricia en el sector público fue de 83 506 y para el año 2018 se pasó a 111 094; no obstante, dicho aumento no redujo la inequidad ${ }^{(12)}$. En el año 2018, la DRHS a nivel nacional fue de 34,5 ; y solo 3 departamentos superaron el valor recomendado por la OMS $(44,5)^{(15)}$. El Ministerio de salud reconoció que esta inequidad distributiva no era solo geográfica sino también socioeconómica precisando, además, que la mayor brecha de disponibilidad de personal de salud ocurría en el primer nivel de atención ${ }^{(16)}$.

En este contexto el gobierno peruano generó normas nacionales para proteger la salud de los RHUS y mejorar su disponibilidad a nivel nacional durante la pandemia de COVID-19. Entre estas normas se consideró que aquellos con condiciones de riesgo para COVID-19 incluido la presencia de comorbilidades o ser adulto mayor, debían ser enviados a aislamiento domiciliario con licencia con goce de haber o para realizar trabajo remoto. Según esto se estimó que $23,7 \%$ de los 215275 profesionales de la salud del sector público registrados a fines de marzo del año 2020, correspondían a la población de riesgo y en su mayoría $(93 \%)^{(17)}$, se encontraban bajo la modalidad de contrato administrativo por servicios a través del régimen laboral 276. Para compensar este déficit de RHUS presencial el gobierno aprobó la contratación de personal de salud bajo un régimen temporal mientras dure la emergencia sanitaria por COVID-19.

Si bien las medidas implementadas teóricamente representan una alternativa de solución para optimizar la situación de los RHUS en el Perú durante la pandemia de COVID-19, a la fecha no hemos encontrado reportes o informes que permitan verificar de forma cuantitativa estos resultados y evaluar si esta respuesta ha sido homogénea alrededor del país. Bajo ese contexto, el presente estudio tiene como objetivo describir los cambios de la densidad de recursos humanos en salud (DRHS) del sector público a nivel nacional y territorial durante el periodo abril-agosto del 2020 en el que se desarrolló la primera fase de la emergencia sanitaria por la pandemia de COVID-19 en el Perú.

\section{MÉTODOS}

\section{Diseño del estudio}

Estudio descriptivo basado en el análisis de datos secundarios usando información proveniente del Registro Nacional de Personal de la Salud (INFORHUS), el cual contiene datos al personal de salud que laboró en los centros de salud del sector público durante la pandemia de COVID-19 en las 25 regiones de Perú entre los meses de abril a agosto de 2020.

\section{Población del estudio}

La población de estudio incluyó al personal de salud del Ministerio de Salud y gobiernos regionales registrados en INFORHUS durante los meses de abril a agosto de 2020. Para fines del cálculo de DRHS se incluyeron únicamente a médicos, enfermeros y obstetras. Para el cálculo del personal de salud en actividad se excluyó a mayores de 65 años, que durante toda la pandemia fueron separados del servicio al público por ser considerados población en riesgo según normativa ${ }^{(18)}$ y a los trabajadores de salud fallecidos por la enfermedad y que por la inmediatez del evento aún no habían sido retirados de INFORHUS. Asimismo, se pusieron restricciones de periodos no 
trabajados al personal de salud que presentó positividad a COVID-19 en pruebas de laboratorio, los cuales tuvieron licencia por descanso médico en el mes de la estimación. La información sobre positividad y fallecimientos por COVID-19 se obtuvo a través de las bases de datos de pruebas rápidas del ministerio de salud (SISCOVID), de pruebas moleculares del Instituto Nacional de Salud (NETLab) y de registros de defunción del Ministerio de Salud (SINADEF).

\section{Variables}

Dentro de las variables incluidas en el estudio se consideraron características sociodemográficas como sexo categorizado en "masculino" y "femenino", edad categorizado en "18-29 años" y "30-59 años" y "60 a más años". Adicionalmente se evaluaron características laborales como el tipo de profesión, categorizado en "medicina", "enfermería" y "obstetricia", régimen laboral incluyendo "régimen 276", "régimen 1057", "régimen 728", y "servicio de terceros", pertenecer o no a una especialidad COVID entre las cuales se incluyeron a medicina interna, radiología, patología, neumología, emergenciología, medicina intensiva, infectología y el mes de registro en INFORHUS durante la cuarentena categorizado en "abril", "mayo", "junio", "julio" y "agosto". Finalmente se recopiló información sobre el tipo de sede de trabajo de los RHUS categorizado como "asistencial" y "administrativa" y el nivel de atención del establecimiento donde trabajaban incluyendo "primer nivel", "segundo nivel" y "tercer nivel".

\section{Análisis estadístico}

Los análisis estadísticos se realizaron a través del software estadístico STATA V.16 y los mapas se hicieron con el software libre QGIS 3.10s. En primer lugar, se realizó un análisis descriptivo a través cuadros y gráficos estadísticos para describir la disponibilidad de médicos, enfermeros y obstetras a nivel nacional teniendo en consideración variables como sexó, edad, régimen laboral, especialidades médicas relacionadas al COVID-19, nivel de atención y tipo de sede.

El análisis de la razón de densidad de recursos humanos en salud (DRHS) se realizó a nivel regional y provincial. El cálculo de DRHS se realizó considerando el total de profesionales de salud en actividad (médicos, enfermeros y obstetras) respecto a la población total asegurada al Seguro Integral de Salud (SIS). La información del SIS se obtuvo a través del portal de la Superintendencia Nacional de Salud (SUSALUD). Con fines comparativos se realizó el cálculo de la variación porcentual de este indicador comparando la DRHS del mes de agosto respecto al mes de abril para cada región y provincia.

\section{Aspectos éticos}

El estudio fue aprobado por el Comité de Ética de la Universidad Peruana Cayetano Heredia. El análisis se realizó integrando la información de las bases de datos mencionadas anteriormente (Figura 1). Esta integración fue coordinada con la Oficina General de Sistemas e Información (OGIS) del Instituto Nacional de Salud. Solo el investigador principal tuvo acceso a identificadores personales y solo durante el periodo de integración. Luego de ello se eliminó cualquier identificador para poder realizar los análisis.

\section{RESULTADOS}

La tabla 1 muestra las características de los RHUS proveniente del INFORHUS por meses. Se detalla la distribución de los profesionales de medicina, enfermería y obstetricia para el cálculo de la DRHS, y los cambios (aumentos y disminuciones) de estas poblaciones por mes. De abril a agosto, se evidenció un aumen-

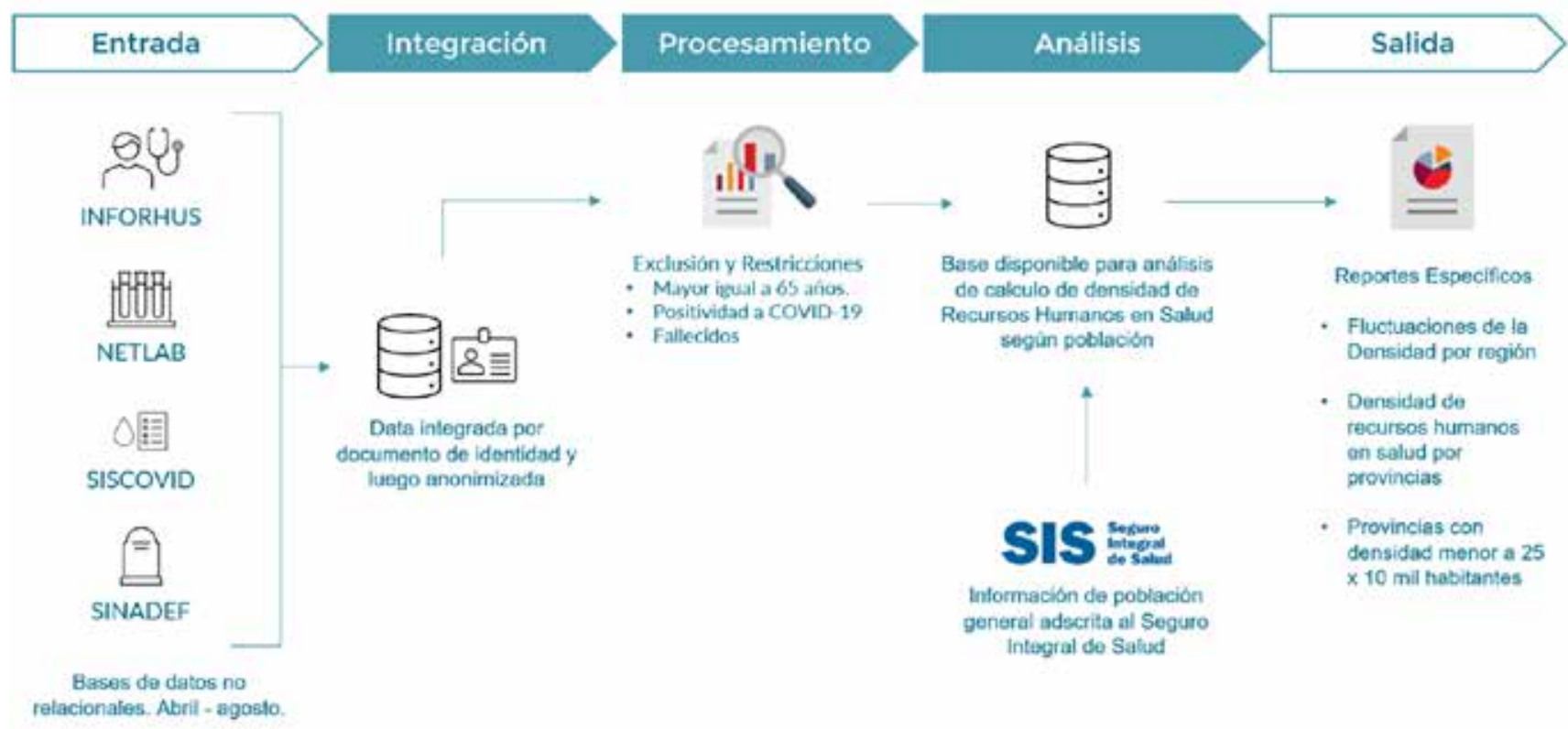

Figura 1. Plan de análisis de la investigación de densidad de recursos humanos en salud en el marco de la pandemia de la COVID-19. Perú, abrilagosto 2020 . 
Tabla 1. Características de los recursos humanos en salud incluidos en el estudio.

\begin{tabular}{|c|c|c|c|c|c|}
\hline \multirow{2}{*}{ Características } & \multicolumn{5}{|c|}{ Recursos Humanos en Salud (RHUS) en sector público por meses } \\
\hline & Abril & Mayo & Junio & Julio & Agosto \\
\hline \multirow{2}{*}{$\begin{array}{l}\text { Total de personal registrado en } \\
\text { INFORHUS }\end{array}$} & 218155 & 233866 & 242467 & 242561 & 249459 \\
\hline & & $(-947 \nabla,+16658 \Delta)$ & $(-5933 \vee,+14534 \Delta)$ & $(-7 \nabla,+101 \Delta)$ & $(-4045 *,+10943 \Delta)$ \\
\hline \multirow[b]{2}{*}{ Total de Médicos (as) } & 27961 & 30630 & 30942 & 31033 & 31651 \\
\hline & & $(-142 \approx,+2811 \Delta)$ & $(-2595 \nabla,+2907 \Delta)$ & $(-3 \approx,+94 \Delta)$ & $(-1196 \nabla,+1814 \Delta)$ \\
\hline Edad & $43,8 \pm 12,3$ años & $43 \pm 12,3$ años & $42,6 \pm 12,5$ años & $42,6 \pm 12,5$ años & $42,6 \pm 12,3$ años \\
\hline \multicolumn{6}{|l|}{ Sexo } \\
\hline Femenino & $11102(39,71 \%)$ & $12408(40,51 \%)$ & $12580(40,66 \%)$ & $12629(40,70 \%)$ & $12364(39,06 \%)$ \\
\hline Masculino & $16859(60,29 \%)$ & $18222(59,49 \%)$ & $18362(59,34 \%)$ & $18404(59,30 \%)$ & $18249(57,66 \%)$ \\
\hline \multicolumn{6}{|l|}{ Nivel de Atención } \\
\hline I nivel & $8771(31,37 \%)$ & $9388(30,65 \%)$ & $10216(33,02 \%)$ & $10220(32,93 \%)$ & $10464(33,06 \%)$ \\
\hline II y III nivel & $18196(65,08 \%)$ & $19823(64,72 \%)$ & $19003(61,41 \%)$ & $19081(61,49 \%)$ & $19227(60,75 \%)$ \\
\hline Sede Administrativa & $925(3,31 \%)$ & $1343(4,38 \%)$ & $1632(5,27 \%)$ & $1641(5,29 \%)$ & $1870(5,91 \%)$ \\
\hline Sin categoría & $69(0,25 \%)$ & $76(0,25 \%)$ & $91(0,29 \%)$ & $91(0,29 \%)$ & $90(0,28 \%)$ \\
\hline \multirow{2}{*}{ Total de Enfermeros (as) } & 37223 & 39623 & 42016 & 42017 & 42775 \\
\hline & & $(-140 \nabla,+2540 \Delta)$ & $(-812 \nabla,+3205 \Delta)$ & $(-0 \nabla,+1 \Delta)$ & $(-766 \nabla,+1524 \Delta)$ \\
\hline Edad & $42,7 \pm 11,4$ años & $42,2 \pm 11,4$ años & $41,6 \pm 11,4$ años & $41,6 \pm 11,4$ años & $41,4 \pm 11,3$ años \\
\hline \multicolumn{6}{|l|}{ Sexo } \\
\hline Femenino & $33295(89,45 \%)$ & $35427(89,41 \%)$ & $37516(89,29 \%)$ & $37517(89,29 \%)$ & $37031(86,57 \%)$ \\
\hline Masculino & $3928(10,55 \%)$ & $4196(10,59 \%)$ & $4500(10,71 \%)$ & $4500(10,71 \%)$ & $4444(10,39 \%)$ \\
\hline \multicolumn{6}{|l|}{ Nivel de Atención } \\
\hline I nivel & $14957(40,18 \%)$ & $15698(39,62 \%)$ & 16961 (40,37\%) & $16961(40,37 \%)$ & $17147(40,09 \%)$ \\
\hline II y III nivel & $20256(54,42 \%)$ & $21250(53,63 \%)$ & $22087(52,57 \%)$ & $22088(52,57 \%)$ & $22570(52,76 \%)$ \\
\hline Sede Administrativa & $1932(5,19 \%)$ & $2590(6,54 \%)$ & $2864(6,82 \%)$ & $2864(6,82 \%)$ & $2953(6,90 \%)$ \\
\hline Sin categoría & $78(0,21 \%)$ & $85(0,21 \%)$ & $104(0,25 \%)$ & $104(0,25 \%)$ & $105(0,25 \%)$ \\
\hline \multirow{2}{*}{ Total de Obstetras } & 16270 & 16965 & 17416 & 17416 & 17588 \\
\hline & & $(-66 \nabla,+761 \Delta)$ & $(-408 v,+859 \Delta)$ & $(-0 \nabla,+0 \Delta)$ & $(-233 \approx,+405 \Delta)$ \\
\hline Edad & $43,3 \pm 10,1$ años & $43 \pm 10,2$ años & $42,6 \pm 10,3$ años & $42,6 \pm 10,3$ años & $42,6 \pm 10,2$ años \\
\hline \multicolumn{6}{|l|}{ Sexo } \\
\hline Femenino & $14803(90,98 \%)$ & 15452 (91,08\%) & $15855(91,04 \%)$ & $15855(91,04 \%)$ & $15732(89,45 \%)$ \\
\hline Masculino & $1467(9,02 \%)$ & $1513(8,92 \%)$ & $1561(8,96 \%)$ & $1561(8,96 \%)$ & $1548(8,80 \%)$ \\
\hline \multicolumn{6}{|l|}{ Nivel de Atención } \\
\hline I nivel & $10945(67,27 \%)$ & $11340(66,84 \%)$ & $11661(66,96 \%)$ & $11661(66,96 \%)$ & $11790(67,03 \%)$ \\
\hline II y III nivel & $4467(27,46 \%)$ & $4684(27,61 \%)$ & $4784(27,47 \%)$ & $4784(27,47 \%)$ & $4783(27,19 \%)$ \\
\hline Sede Administrativa & $845(5,19 \%)$ & $927(5,46 \%)$ & $958(5,50 \%)$ & $958(5,50 \%)$ & $1000(5,69 \%)$ \\
\hline Sin categoría & $13(0,08 \%)$ & $14(0,08 \%)$ & $13(0,07 \%)$ & $13(0,07 \%)$ & $15(0,09 \%)$ \\
\hline
\end{tabular}

- Disminución de RHUS respecto al mes previo, $\mathbf{\Delta}$ Aumento de RHUS respecto al mes previo

to progresivo de las poblaciones estudiadas, siendo en el mes de mayo ocurrió el máximo incremento. En los tres grupos profesionales se encontró una edad promedio inicial de entre 42 y 43 años, la cual disminuyó a 41 y 42 años para el mes de agosto. Todos los meses mostraron una mayor proporción de profesionales médicos y enfermeros laborando en el segundo nivel de atención, seguido del primer nivel atención y finalmente en sedes administrativas. En el caso de los profesionales médicos, la proporción de recursos humanos que laboraban en el primer nivel de atención aumentó de abril a agosto. En los profesionales obstetras, la mayor proporción se encontró siempre en el primer nivel de atención, la 


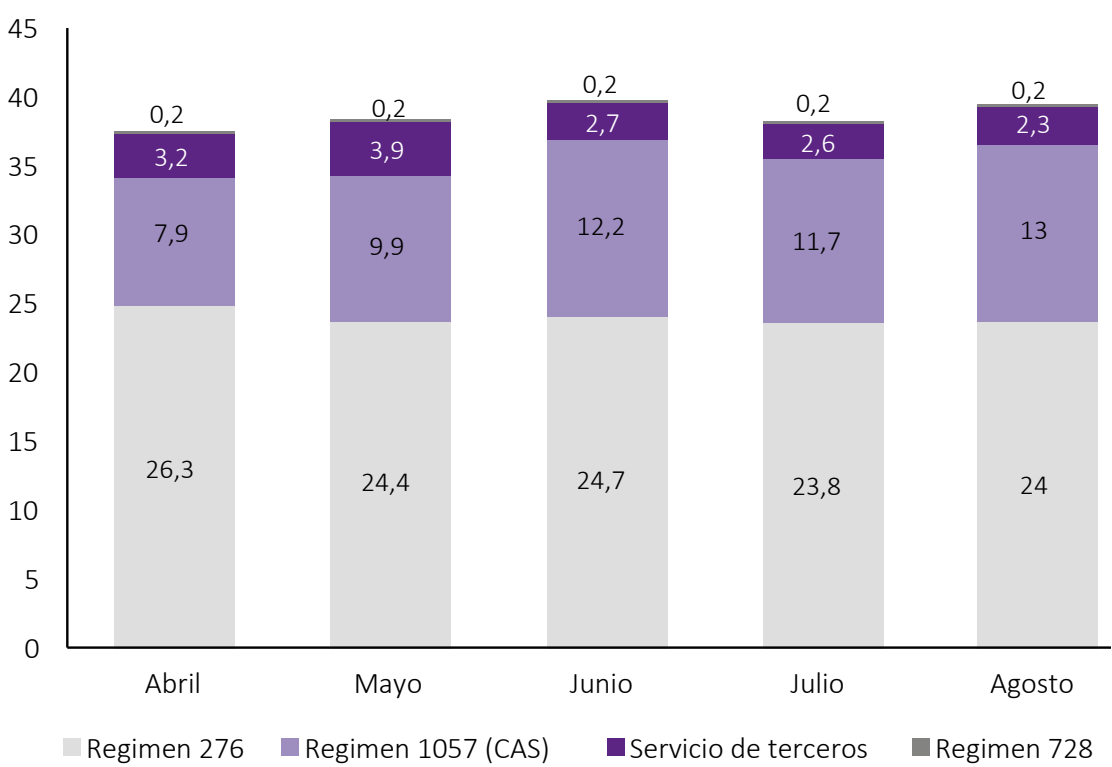

Figura 2. Razón de densidad de recursos humanos en salud por régimen laboral, abril - agosto 2020.

cual también mostro un aumento desde abril a agosto.

Al realizar el análisis de densidad de recursos humanos en salud, se encontró

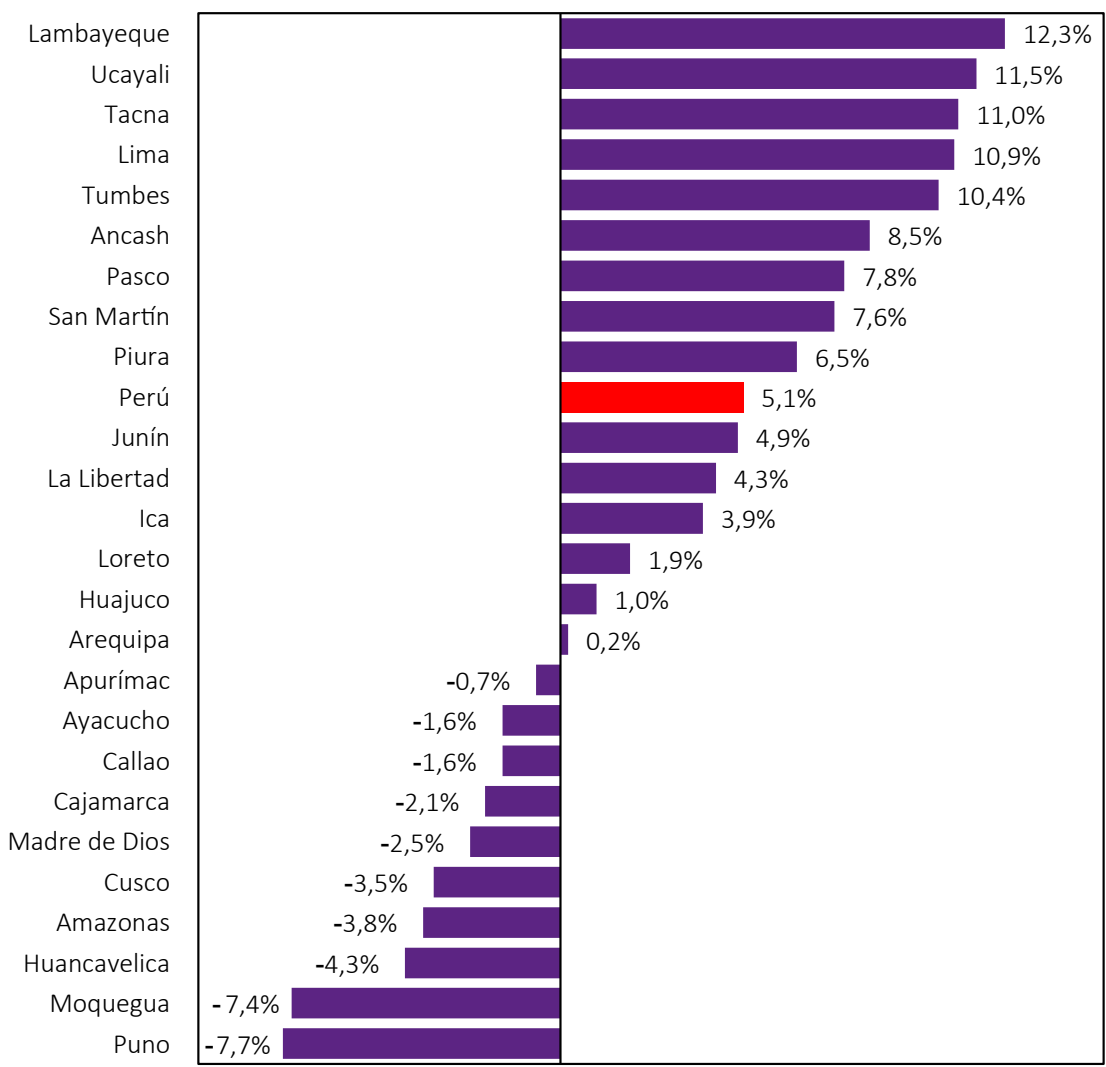

Figura 3. Variación porcentual de la densidad de RHUS por regiones. Perú, abril - agosto 2020. vo de $5,1 \%$. Como se aprecia en la figura 2, el impacto de la pandemia COVID-19 sobre el personal estable de MINSA y los gobiernos regionales (régimen laboral del Decreto Legislativo $\mathrm{N}^{\circ} 276$ ) tuvo una reducción de DRHS de 26,3 a 24,4 x 10 000 en un solo mes manteniéndose con una variación muy ligera hasta el final del periodo analizado. La DRHS que laboran bajo modalidad temporal (CAS) incrementó constantemente de 7,9 a $13 \times 10$ 000 al final de periodo observado. A nivel provincial se han reportado 68 provincias con DRHS mayores o iguales a 40 x 10000 habitantes, y 55 provincias ( $28 \%$ ) con una DRHS menor de $25 \times 10000$ habitantes).

Respecto a la DHRS en las diversas regiones, se presentaron diversas dinámicas de variación. La región Lima (capital de Perú), presentó una reducción en el personal de salud estable, pasando de una DHRS de 31,2 a 27,7 x 10000 habitantes, mientras que casi la DHRS en personal CAS se incrementó en 149,8\% (Figura 3). Por otro lado, las regiones como Lambayeque $(12,3 \%)$, Ucayali $(11,5 \%)$ y Tacna (11\%) fueron las que incrementaron más su densidad de recursos humanos, mientras que diez regiones presentaron una disminución del indicador para el periodo de estudio, como las regiones de Puno $(-7,7 \%)$, Moquegua $(-7,4 \%)$ y Huancavelica (-4,3\%).

Cuando se observan los cambios de la DRHS a nivel de las provincias (Figura 4), se observa que 87 de las 196 provincias presentan un incremento promedio de $8,2 \%$ (DE $\pm 7,8 \%$ ). Dentro de las provincias con mayor porcentaje de incremento se encontraron: Chupaca (Junín) +52,3\%; Huarochirí (Lima) $+26,2 \%$, Casma (Ancash) $+25,4 \%$, Mariscal Ramon Castilla (Loreto) $+20,7 \%$, Islay (Arequipa) $+20,7 \%$, Lambayeque (Lambayeque) $+18,8 \%$, Carabaya (Puno) $+18,2 \%$, San Martín (San Martín) $+18,1 \%$; Talara (Piura) $+17,1 \%$ y Zarumilla (Tumbes) $+16,4 \%$, entre otros.

Por el contrario, en 105 provincias se observó una reducción de la DRHS; en promedio un $8,2 \% \%$ (DE $\pm 6,2 \%$ ). Dentro de las provincias con mayor porcentaje de disminución se encontraron: Ocros (Ancash) -28,3\%, Manu (Madre de Dios) $-27,6 \%$, Tahuamanu (Madre de Dios) -24,3, Purus (Ucayali) -21,5\%, Urubam- 


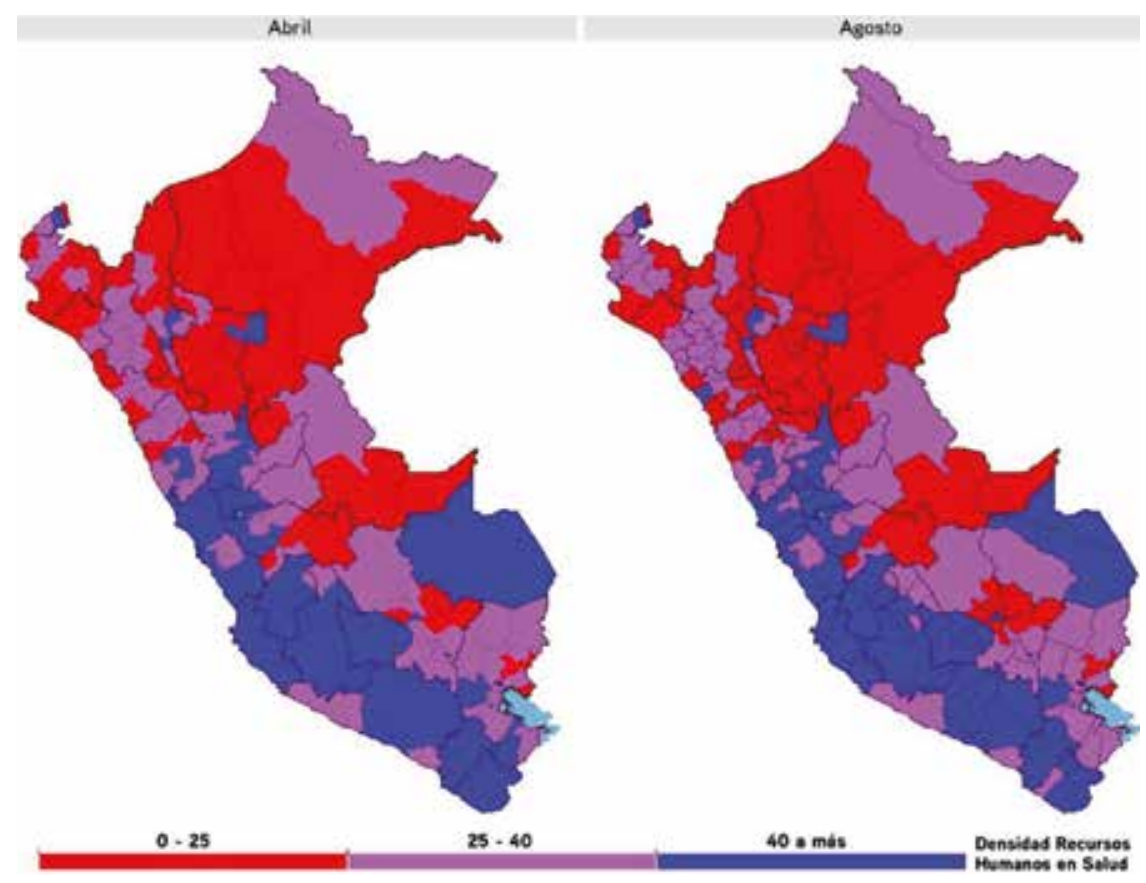

Figura 4. Razón de densidad de recursos humanos según provincia. Perú, abril-agosto 2020.

ba (Cuzco) $-21,4 \%$, San Antonio de Putina (Puno) $-20,6 \%$, Paucartambo (Cuzco) $-20,3 \%$, Pallasca (Ancash) $-17,7 \%$, Tarata (Tacna) $-16,8 \%$, San Román (Puno) $-15,8 \%$, entre otros. Solo en 5 provincias no se observaron cambios en la DRHS.

Cuando se analizan los cambios de DRHS según niveles de atención (Figura $5)$, se observaron variaciones menores a $1 \times 1000$. No obstante, se encuentran

respuestas diferenciadas en algunos territorios. Por ejemplo, algunas regiones presentaron mayores incrementos en la densidad de recursos humanos en el primer nivel de atención, como Lambayeque $(+18,4 \%)$, Tumbes $(+10,9 \%)$ y Pasco $(+10,2 \%)$. Otras regiones concentraron el incremento de la densidad de recursos humanos en el segundo nivel de atención, como Tacna (+39\%), Lima (+23,6), Huánu-

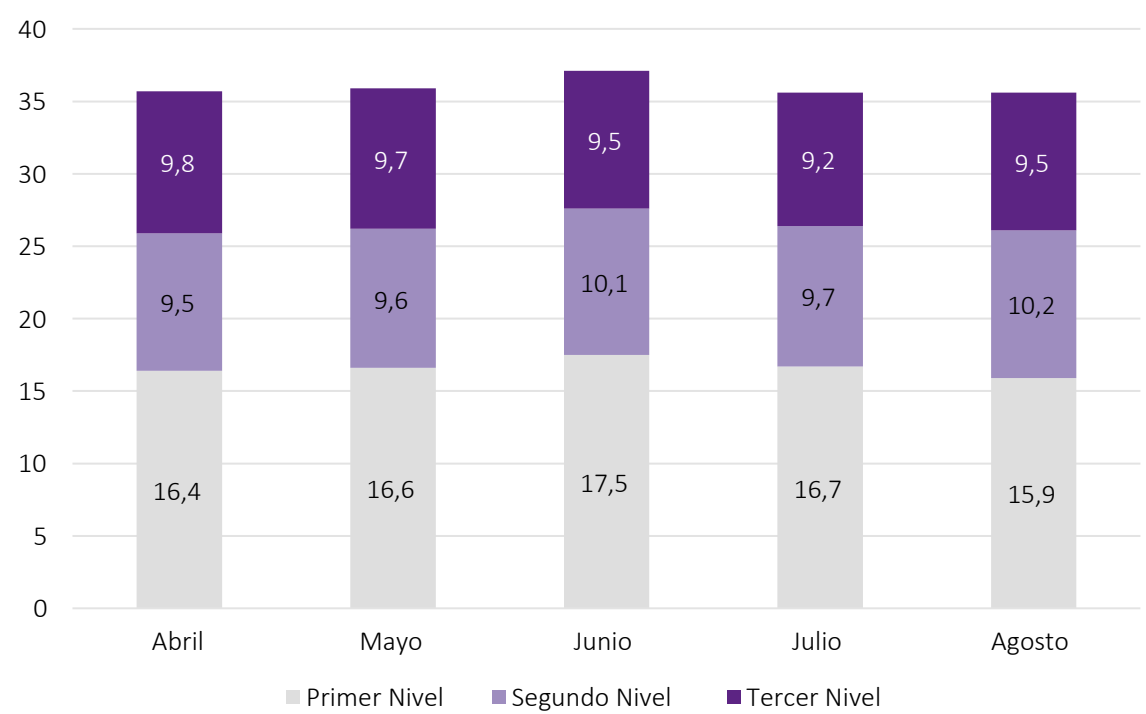

Figura 5. Razón de densidad de recursos humanos por nivel de atención. Perú, abril- agosto 2020. co $(+21,3 \%)$ y Pasco $(+21,1 \%)$. Respecto al tercer nivel de atención, Arequipa (+12\%), La Libertad $(+7,5 \%)$ y Junín $(+4,1 \%)$ presentaron los mayores incrementos.

\section{DISCUSIÓN}

Los hallazgos de nuestro estudio muestran que la situación de la disponibilidad los RHUS durante el primer contexto de cuarentena por la pandemia de COVID-19 en el Perú, tuvo un incremento en la DRHS a nivel nacional de 5,1\% entre los meses de abril a agosto de 2020. Sin embargo, este incremento presentó diversas variaciones alrededor de las regiones del país presentando por un lado un incremento de este indicador de 10 a $12 \%$ en regiones como Lambayeque, Ucayali, Tacna y Lima, mientras que Moquegua y Puno mostró una reducción de hasta $-7,7 \%$. Además, estas variaciones se mostraron aún más acentuadas en el análisis a nivel provincial y por niveles de atención de los establecimientos de salud.

La principal explicación para el incremento de la DRHS visto a nivel nacional y en algunas regiones del país puede venir de la expansión de contratos temporales (contratos CAS) en los centros de salud del MINSA y el estímulo económico brindado por el gobierno para atender la emergencia. Sin embargo, a pesar facilitar la obtención de personal de forma inmediata, la implementación de esta estrategia no ha sido suficiente para evitar el colapso del sistema de salud en diversos territorios a nivel nacional, especialmente en aquellos con inequidades previas a la pandemia en relación a este recurso ${ }^{(12,19)}$. Esto indica que la aplicación de contratos de corto plazo resultaría ineficaz como única estrategia en contextos donde se cuenta con una reducida oferta de RHUS ${ }^{(20)}$.

Por otro lado, nuestros hallazgos muestran que durante la pandemia se ha incrementado la inequidad en materia de RHUS a nivel regional y provincial. Esto indica la existencia de diversos sectores en donde no solo persiste un déficit de este recurso, sino que se ha agravado durante la cuarentena generando mayores limitaciones en el despliegue de la labor 
asistencial para la atención de pacientes infectados y el desarrollo de intervenciones esenciales en salud pública, incluyendo, por ejemplo, la realización de pruebas diagnósticas o aplicación de vacunas para el COVID-19. Ante ello, resulta urgente el despliegue de estrategias que permitan compensar la disponibilidad de RHUS en estos sectores dada las evidencias que señalan un mayor impacto del COVID en las poblaciones peruanas con deficiencias en este recurso ${ }^{(21,22)}$.

Dada la situación desigual a nivel regional y provincial respecto al DRHS recalca la necesidad de implementar estrategias diferenciadas que permitan optimizar y/o compensar la situación de RHUS en cada uno de estos sectores de acuerdo con sus necesidades. Por un lado, en sectores con un adecuado nivel de DRHS resultaría provechoso implementar estrategias para lograr una mayor eficiencia de la labor de los RHUS disponibles y desarrollar intervenciones de control del COVID basadas en la acción del primer nivel de atención. Mientras tanto los sectores más desfavorecidos podrían beneficiarse de la implementación de estrategias combinadas, basadas en acciones comunitarias y equipos de respuesta móvil.

Es importante explicar la metodología empleada en el presente estudio para la medición de la DRHS y evaluación de los cambios de este indicador en un escenario tan dinámico como la epidemia de COVID-19 en el Perú. Los criterios de exclusión elegidos buscaron establecer un número que se acerque lo mejor posible a la disponibilidad real de personal en los servicios, descontando a los trabajadores afectados por la epidemia. También debemos señalar la ventaja que significa tomar como referencia la población del Seguro Integral de Salud, construida a partir de registros individuales, que nos plantea un rango de mayor exactitud, que las poblaciones de referencia estimadas por el INEI, metodología de estimación que ya ha sido aplicada anteriormente en nuestro medio ${ }^{(23)}$.

Por otro lado, el presente estudio cuenta con algunas limitaciones a mencionar. En primer lugar, al tratarse de un análisis secundario de base de datos, los resultados están supeditados a las características de la información disponible. Si bien la DHRS es un indicador de constante cambio, el análisis fue realizado de forma mensual debido a que la información verificada de INFORHUS presentaba cortes de actualización con esta frecuencia. Asimismo, se utilizó el supuesto de que los periodos no laborales en los RHUS con resultado positivo a COVID-19 fueron los mismos para todos los casos ya que no se tuvo acceso a información sobre su gravedad o tiempo de enfermedad. Tampoco se obtuvo información que permitiera constatar si los profesionales que se encontraban con licencia realizaron teletrabajo o no. Finalmente, la DRHS en salud se calculó basándose solo en médicos, enfermeras y obstetras tal y como se sugiere en publicaciones de la OMS. Sin embargo, es posible que durante la pandemia la prestación de servicios críticos no se haya limitado únicamente a esas profesiones. Aun con estas limitaciones, consideramos que los cálculos realizados en el presente estudio respecto a la DHRS representan una adecuada aproximación de la la disponibilidad de RHUS en actividad durante el primer periodo de cuarentena en el Perú.

El desafío para el sistema de salud en el mediano plazo es enorme, porque en la práctica, se requiere dimensionar un sistema de salud con capacidad para sostener la respuesta al COVID-19, incluyendo programas anuales de vacunación universal, así como retomar las necesidades de la población no atendidas durante el último año, lo que crea una enorme presión sobre un sistema de salud que va a tener que escalar su oferta, tanto en las grandes ciudades como en los territorios. En ese escenario, la necesidad de priorizar la protección de los trabajadores de salud, fortalecer las acciones de cuidado, recuperación e reinserción del personal afectado por el COVID-19 y la ampliación de la formación y reclutamiento de nuevos profesionales de la salud, en un elemento central en la sostenibilidad del sistema de salud peruano de cara a la actual crisis sanitaria ${ }^{(24)}$.

\section{Conclusiones y recomendaciones}

Los resultados en nuestro estudio muestran que en el Perú la disponibilidad de RHUS presentó un leve incremento durante el periodo de cuarentena por COVID-19; sin embargo, estos cambios no se han visto reflejados a nivel regional, en donde persisten o han agravado su situación respecto a la disponibilidad de este recurso. Esta situación supone un desafío importante para el sistema de salud local, situación que se ha visto incluso países de altos ingresos quienes han tenido dificultades para enfrentar adecuadamente la pandemia y cuyos sistemas de salud se han debilitado o colapsado ${ }^{(25)}$. Esta situación implica tener una mirada integral de la complejidad del despliegue de los recursos humanos en salud en el contexto de la actual fragmentación del sistema de salud peruano especialmente en este escenario de crisis sanitaria.

Dado el contexto y los resultados encontrados en nuestro estudio, recalcamos la necesidad de realizar mayores estudios que incorporen el análisis de la DRHS de otros subsistemas como el Seguro Social del Perú y las Fuerzas Armadas y Policiales a fin de desarrollar una política pública de fortalecimiento de los recursos humanos de alcance nacional y que optimice la distribución de los limitados recursos disponibles. Adicionalmente, sugerimos el establecimiento de un mecanismo de interoperabilidad entre las bases de datos empleadas en el presente estudio, con miras a establecer un sistema de seguimiento de la evolución de las variables analizadas, permitiendo que la expansión de la disponibilidad de RHUS se pueda monitorear para velar por la equidad del sistema a través de la respuesta diferenciada en función del impacto, también diferenciado, de la pandemia en territorios departamentales o provinciales del país.

\section{REFERENCIAS BIBLIOGRÁFICAS}

1. Organización Panamericana de la Salud. Manual de medición y monitoreo de indicadores de las metas regionales de recursos humanos para la salud: un compromiso compartido [Internet]. Washington: OPS; 2011 [citado 18 de febrero de 2021]. Disponible en: https://www. paho.org/hq/index.php?option=com_content\& view $=$ article $\& i d=5696: 2011$ - manual-medicionmonitoreo-indicadores-metas-regionales-recursoshumanos\&ltemid $=42273 \&$ lang $=$ es

2. Organización Panamericana de la Salud J. $29^{\mathrm{a}}$ Conferencia Sanitaria Panamericana. Sesión del Comité 
Regional de la OMS para las Américas. Estrategia de Recursos Humanos para el Acceso Universal a la Salud y la Cobertura Universal de Salud. [Internet]. Washington, D.C., EUA: OPS; 2017 jul [citado 18 de febrero de 2021]. Disponible en: https://www. paho.org/hq/index.php?option=com_content\&vie w=article\&id=13497:29th-pan-american-sanitaryconference\&ltemid $=2105 \&$ lang $=e s$

3. Walton-Roberts M. COVID-19 and Global Human Health Resources. 2020

4. Corona T, Castañón-González JA, Clark-Peralta P, Garcia-Peña C, Guevara-Guzmán R, DomínguezCherit G, et al. Position paper: Impact on medica and health personnel in the SARS-CoV-2 pandemic. Gac Med Mex. 2020;156(5):478-80. DOI: 10.24875 GMM.M20000432

5. Carmassi C, Foghi C, Dell'Oste V, Cordone A, BerteIloni CA, Bui E, et al. PTSD symptoms in healthcare workers facing the three coronavirus outbreaks: What can we expect after the COVID-19 pandemic Psychiatry Res. 2020;292:113312. DOI: 10.1016/j. psychres.2020.113312

6. Kingston AM. Break the Silence: Physician Suicide in the Time of COVID-19. Mo Med. 2020;117(5):426-9.

7. Teksin G, Uluyol OB, Onur OS, Teksin MG, Ozdemi HM. Stigma-related Factors and their Effects on Health-care Workers during COVID-19 Pandemics in Turkey: A Multicenter Study. Med Bull Sisl Etfal Hosp. 2020;54(3):281-90. DOI: 10.14744/ SEMB.2020.02800

8. Mesa de Concertación para la Lucha Contra la Pobreza. Informe Nacional sobre el Impacto de Covid-19 en las Dimensiones Económica, Social y en Salud en el Perú [Internet]. Lima, Perú.; 2020 jul [citado 18 de febrero de 2021] p. 26-33. Report No.: Reporte N¹-2020-SC/Grupo de Salud-MCLCP. Disponible en: https://www.mesadeconcertacion org.pe/noticias/mesa-nacional/informe-nacionalsobre-el-impacto-del-covid-19-en-las-dimensioneseconomica-social-y-en-salud-en-el-peru
9. Chafloque-Vásquez RA, Pampa-Espinoza L, Celis Salinas JC, Chafloque-Vásquez RA, PampaEspinoza L, Celis Salinas JC. Seroprevalencia de COVID-19 en trabajadores de un hospital de la Amazonia peruana. Acta Médica Peru. 2020;37(3):390-2. DOI: https://doi.org/10.35663/amp.2020.373.1050

10. Arroyo J. Los sistemas descentrados de recursos humanos en salud: el caso del Perú, 1990-2005. Ciênc Saúde Coletiva. 2006;11(4):1063-72.

11. Alcalde-Rabanal JE, Lazo-González O, Nigenda G. Sistema de salud de Perú. Salud Pública México. 2011;53:s243-54.

12. Moscoso Rojas B, Huamán Angulo L, Núñez Vergara M, Llamosas Felix E, Perez W. Inequidad en la distribución de recursos humanos en los establecimientos del Ministerio de Salud de cuatro regiones del Perú. An Fac Med. 2015;76(SPE):35-40. DOI: https://doi.org/10.15381/anales.v76i0.10968

13. Gutierrez H, Shewade A, Dai M, Mendoza-Arana P, Gómez-Dantés O, Jain N, et al. Health Care Coverage Decision Making in Low- and MiddleIncome Countries: Experiences from 25 Coverage Schemes. Popul Health Manag. 2015;18(4):265-71. DOI: 10.1089/pop.2014.0099

14. García Cabrera HE, Díaz Urteaga P, Ávila Chávez D, Cuzco Ruiz MZ. La Reforma del Sector Salud y los recursos humanos en salud. An Fac Med. 2015;76(SPE):7-26. DOI: https://doi.org/10.15381/ anales.v76i0.10966

15. Dirección General de Personal de la Salud. Información de Recursos Humanos en el Sector Salud Perú - 2018 [Internet]. Perú: MINSA -DIGEP.; 2018 [citado 18 de febrero de 2021]. Disponible en: https://drive.minsa.gob.pe/s/SPSCikgyzBfNssx

16. Ministerio de Salud. Lineamientos para a vigilancia de la salud de los trabajadores con riesgo de exposición a COVID-19 [Internet]. Resolución Ministerial N²39-2020-MINSA | Gobierno del Perú abr 29, 2020. Disponible en: https://www.gob.pe/institucion/ minsa/normas-legales/542920-239-2020-minsa
17. Dirección General de Personal de la Salud. DIGEP OBSERVATORIO [Internet]. 2020 [citado 21 de febrero de 2021]. Disponible en: http://digep.minsa. gob.pe/

18. Presidencia del Consejo de Ministros. Decreto Supremo que prorroga el Estado de Emergencia Nacional por las graves circunstancias que afectan la vida de la Nación a consecuencia del COVID-19 y establece otras disposiciones [Internet]. Decreto Supremo Nº 094-2020-PCM. Disponible en: https://www.gob.pe/institucion/pcm/normas legales/584231-094-2020-pcm

19. Mendoza-Arana PJ, Rio GR-D, Gutiérrez-Villafuerte C, Sanabria-Montáñez C. El proceso de reforma del sector salud en Perú. Rev Panam Salud Pública. 2018:42:e74. DOI: 10.26633/RPSP.2018.74

20. Arana PM. Aspectos Económicos de la Formación de los Profesionales de la Salud. An Fac Med. 1998;59(3):244-8. DOI: https://doi.org/10.15381/ anales.v59i3.4627

21. Gianella C, Gideon J, Romero MJ. What does COVID-19 tell us about the Peruvian health system? Can J Dev Stud Can Détudes Dév. 2020;42(1-2): 55-67. DOI: 10.1080/02255189.2020.1843009

22. Montag D, Barboza M, Cauper L, Brehaut I, Alva I, Bennett A, et al. Healthcare of Indigenous Amazonian Peoples in response to COVID-19: marginality, discrimination and revaluation of ancestral knowledge in Ucayali, Peru. BMJ Glob Health. 2021;6(1): e004479. DOI: 10.1136/bmjgh-2020-004479

23. Garcia AEO. Desigualdad en la distribución de médicos en el Perú. Rev Cuba Salud Pública. 2020;47(1).

24. The Lancet. Health and care workers are owed a better future. Lancet. 2021;397(10272):347. DOI: 10.1016/S0140-6736(21)00179-3

25. Sundararaman T. Health systems preparedness for COVID-19 pandemic. Indian J Public Health. 2020;64(6):91-93. DOI: 10.4103/ijph.IJPH_507_20 